\title{
VECTOR-LIKE FERMION AND STANDARD HIGGS PRODUCTION AT HADRON COLLIDERS*
}

\author{
F. del AGUILA ${ }^{\mathrm{a}}$, Ll. AMETLLER ${ }^{\mathrm{b}}$, G.L. KANE ${ }^{\mathrm{c}}$ and J. VIDAL ${ }^{\mathrm{d}}$ \\ ${ }^{a}$ Departament de Física Teòrica, Universitat Autònoma de Barcelona, 08193 Bellaterra \\ (Barcelona), Spain \\ ${ }^{\mathrm{b}}$ Departament de Física i Enginyeria Nuclear, EUPV, Universitat Politècnica de Catalunya, \\ Q8800 Vilanova (Burcelona), Spain \\ ${ }^{\mathrm{c}}$ Randall Physics Laboratory, University of Michigan, Ann Arbor, MI 48109, USA \\ ${ }^{\mathrm{d}}$ Departament de Física Teòrica, Univ. de València, and IFIC. Univ. de València-CSIC, 46100 \\ Burjassot (València), Spain
}

Received 21 September 1989

\begin{abstract}
Vector-like fermions are characterized by large neutral current decay rates, in particular into Higgs bosons. If they exist, their clear signals at hadron colliders open a window to Higgs detection, especially to the intermediate Higgs mass region. We discuss in some detail rates and signaturcs for simple cases.
\end{abstract}

\section{Introduction}

Vector-like fermions [1-14] are characterized by having their left- and righthanded components transforming in the same way under the symmetry group of the theory. For this reason their mass terms, $\bar{\Psi}_{\mathrm{L}} \Psi_{\mathrm{R}}$, are not forbidden by any symmetry. As a consequence their masses are unbounded and they decouple when these are taken to infinity [2]. This explains why they are essentially unconstrained by present phenomenology, except for the bounds resulting from production limits [3]. On similar grounds they can often contribute to new effects without upsetting existing data.

The standard model may not need vector-like fermions, but by the same argument they are among the few particles which can naturally exist near the electroweak scale and have no mandatory implications on the low-energy phenomenology (although they may accommodate better $C P$ violation, ... [8-10]). It is important to note that although this is surprising (because they are so apparently unconstrained) their properties are to a large extent fixed! In particular, their decays are known to some extent, and if they are produced, their detection can be (readily) accomplished, and their properties stated [11]. It is important to note that vector-like fermions

* Research supported in part by the US DOE by CICYT and by the Spain-U.S. committee. 
decay by exchange of standard electroweak gauge and Higgs bosons, $W^{ \pm}, Z, H$, with all three being comparable in size! Thus, vector-like fermions may transform a collider into a Higgs factory [12]. The subject of this paper is the production and decay of heavy vector-like fermions at hadron colliders, with special attention being paid to the Higgs decay channel. In particular we will find that if a vector-like fermion with mass $M_{\mathrm{F}} \leqslant 1 \mathrm{TeV}$ exists, a Higgs in the intermediate mass region $M_{\mathrm{H}}<2 M_{\mathrm{W}}$ and decaying into $\mathrm{q} \overline{\mathrm{q}}$ cannot escape detection as the SSC.

It is easy to understand the relevant physics. Assume we add a vector-like quark to the standard model. It will be stable in the absence of mixing with the known fermions. This tells us that decay rates, and in general the physics of this new fermion, are proportional to this mixing. Since the mixing results from the Yukawa couplings giving masses to the standard fermions, that is to say from the Higgs-fermion vertices, a dominant source of vector-like fermion decays is through Higgs exchange. Moreover, these vertices are proportional to the corresponding gauge boson-fermion vertices, because the latter themselves arise from the interplay of current- and mass-fermion eigenstates which result from the mass generation itself and thus from the same Yukawa couplings. The arbitrariness is (as always) due to the mass of the new particle being unknown. (The $\mathrm{Z}$ couplings - like the $\mathrm{H}$ ones - imply tree level flavour changing neutral currents. Many consequences have been discussed elsewhere [1-14].)

The main point of the paper, the large branching ratio of a new vector-like fermion into a Higgs, was presented in ref. [12] for the case of a new down quark at the Tevatron. Here we present a more detailed discussion with emphasis on the backgrounds for the different colliders and for the different extra vector-like fermions in the $27+\overline{27}$ representation of $\mathrm{E}_{6}$ [15]. Vector-like fermion production at $\mathrm{e}^{+} \mathrm{e}^{-}$colliders was discussed elsewhere [13], taking into consideration the possibility of an extra neutral gauge boson $Z^{\prime}$. New vector-like lepton doublets were previously considered at the SSC [14]. In all cases only vector boson decays were studied. Cross sections are expected to be small at HERA for new vector-like fermions would have to be produced through their mixing with standard fermions.

In sect. 2 we present the simplest possible cases. We add to the standard model first one extra vector-like fermion with the quantum numbers of a down singlet $D$ quark, then an up down quark doublet $\left(\begin{array}{l}U \\ D\end{array}\right)$, and then an up singlet $U$ quark, as well as a singlet charged lepton $E$ and a neutral charged lepton doublet $\left(\begin{array}{l}N \\ E\end{array}\right)$ (a heavy neutral singlet $\mathrm{N}$ would be produced through its mixing with standard leptons, and the corresponding cross section is negligible). The relevant lagrangian is spelled out. The production cross sections are similar to those of the standard fermions, whereas the decay rates are dictated by the different particle masses and the corresponding kinematics. In sect. 3 we discuss the signatures and backgrounds at hadron colliders (we work at the parton level). Finally sect. 4 is devoted to generalizations and conclusions. 


\section{Production rates and branching ratios}

The production of vector-like fermions is most efficient through the dominant standard family diagonal couplings to gluons and electroweak gauge bosons, with the difference that the $W_{\mu}^{ \pm}$and $Z_{\mu}$ couplings are vector-like, they couple to $\bar{\Psi} \gamma^{\mu} \Psi$. The decays are governed by the leading mixing terms. In table 1 we give the lagrangian terms relevant for the different cases (in obvious notation). They are well known [3] and the terms of present interest are those involving the standard Higgs $H$. Let us discuss those involving a new down quark singlet $D$, already discussed in refs. $[9,12]$. We can choose a current eigenstate basis where the down mass matrix is written as

$$
\begin{aligned}
& d_{\mathrm{R}} \quad D_{\mathrm{R}} \\
& \mathscr{M}_{\mathrm{d}}=\overline{\bar{d}}_{\mathrm{L}}\left(\begin{array}{llll}
m_{\mathrm{d}} & & & m_{1}^{\prime} \\
& m_{\mathrm{s}} & & m_{2}^{\prime} \\
& & m_{\mathrm{b}} & m_{3}^{\prime} \\
& & M_{\mathrm{Q}}
\end{array}\right)
\end{aligned}
$$

and zero elsewhere. $M_{\mathrm{Q}}$ is the mass of the new vector-like down quark and $m_{i}^{\prime}$ give the mixing. The decay terms in table 1 result from working out the lagrangian in the mass eigenstates to first order in $m_{i}^{\prime} / M \ll 1$. (The Cabibbo-Kobayashi-Maskawa matrix, $C$, appears when the up quarks are taken into account.) For the other cases we proceed in a similar way, the difference being the proper initial matrices. In particular, for an extra quark doublet they read

$$
\begin{aligned}
& u_{\mathrm{R}} \quad U_{\mathrm{R}} \\
& \mathscr{M}_{\mathrm{u}}=\bar{u}_{\mathrm{L}}\left(\begin{array}{c}
\Delta_{i j} \\
\bar{U}_{\mathrm{L}} m_{2} m_{3} M
\end{array}\right), \\
& \Delta=C^{\dagger}\left(\begin{array}{lll}
m_{\mathrm{u}} & & \\
& m_{\mathrm{c}} & \\
& & m_{\mathbf{1}}
\end{array}\right) C^{\prime}, \\
& d_{\mathrm{R}} \quad D_{\mathrm{R}}
\end{aligned}
$$

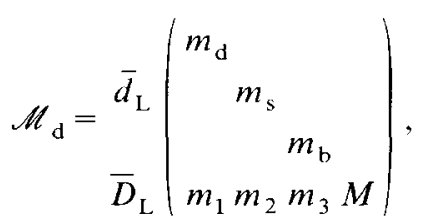


TABLE 1

Relevant lagrangian terms for vector-like fermion production and decay

New vector-like

fermion

Dominant production terms

$\mathrm{D}$

$\left(\begin{array}{l}\mathrm{U} \\ \mathrm{D}\end{array}\right)$

$\mathrm{U}$

$\mathrm{E}$

$\left(\begin{array}{l}N \\ E\end{array}\right)$
$\mathscr{L}_{\mathrm{QCD}}=-i g_{s} A_{a \mu} \bar{D} T^{a} \gamma^{\mu} D$

$\mathscr{L}_{\mathrm{QCD}}=-i g_{s} A_{a \mu}\left(\bar{U} T^{a} \gamma^{\mu} U+\bar{D} T^{a} \gamma^{\mu} D\right)$

$\mathscr{L}_{\mathrm{QCD}}=-i g_{s} A_{a \mu} \bar{U} T^{a} \gamma^{\mu} U$

$\mathscr{L}_{\gamma+\mathrm{Z}}=e A_{\mu} J_{\mathrm{EM}}^{\mu}-\frac{e s_{\mathrm{W}}}{c_{\mathrm{W}}} Z_{\mu} J_{\mathrm{EM}}^{\mu} ;$

$$
J_{\mathrm{EM}}^{\mu}=-\bar{E} \gamma^{\mu} E
$$

$$
\mathscr{L}_{\gamma+\mathrm{W}^{ \pm}+\mathrm{Z}}=e A_{\mu} J_{\mathrm{EM}}^{\mu}
$$

$$
\begin{aligned}
& +\frac{g_{2}}{2 c_{\mathrm{w}}} Z_{\mu}\left(\bar{N} \gamma^{\mu} N-\bar{E} \gamma^{\mu} E-2 s_{\mathrm{W}}^{2} J_{\mathrm{EM}}^{\mu}\right) \\
& +\frac{g_{2}}{\sqrt{2}} W_{\mu}^{+} \bar{N} \gamma^{\mu} E+\text { h.c.; } \quad J_{\mathrm{EM}}^{\mu}=-\bar{E} \gamma^{\mu} E
\end{aligned}
$$

Dominant decay terms

D

$$
\begin{aligned}
\mathscr{L}= & \left(\frac{g_{2}}{\sqrt{2}} W_{\mu}^{+} \bar{u}_{\mathrm{L} i} \gamma^{\mu} C_{i j} \frac{m_{j}^{\prime}}{M_{\mathrm{Q}}} D_{\mathrm{L}}\right. \\
& \left.-\frac{g_{2}}{2 c_{\mathrm{W}}} Z_{\mu} \bar{d}_{\mathrm{L} i} \gamma^{\mu} \frac{m_{i}^{\prime}}{M_{\mathrm{Q}}} D_{\mathrm{L}}-H \bar{d}_{\mathrm{L} i} \frac{m_{i}^{\prime}}{v} D_{\mathrm{R}}\right)+ \text { h.c. }
\end{aligned}
$$

$$
\begin{aligned}
\mathscr{L}= & \left(-\frac{g_{2}}{\sqrt{2}} W_{\mu}^{+}\left[\bar{U}_{\mathrm{R}} \gamma^{\mu} \frac{m_{j}}{M_{\mathrm{Q}}} d_{\mathrm{R} j}+\bar{u}_{\mathrm{R} j} \gamma^{\mu} C_{j i}^{\prime} \frac{m_{i}^{*}}{M_{\mathrm{Q}}} D_{\mathrm{R}}\right]\right. \\
& -\frac{g_{2}}{2 c_{\mathrm{W}}} \mathrm{Z}_{\mu}\left[\bar{U}_{\mathrm{R}} \gamma^{\mu} \frac{m_{i}}{M_{\mathrm{Q}}} C_{i k}^{\prime+} u_{\mathrm{R} k}-\bar{d}_{\mathrm{R} j} \gamma^{\mu} \frac{m_{j}^{*}}{M_{\mathrm{Q}}} D_{\mathrm{R}}\right] \\
& \left.-H\left[\bar{U}_{\mathrm{L}} \frac{m_{i}}{v} C_{i k}^{\prime} u_{\mathrm{R} k}+\bar{d}_{\mathrm{R} j} \frac{m_{j}^{*}}{v} D_{\mathrm{L}}\right]\right)+ \text { h.c. }
\end{aligned}
$$

$\mathrm{U}$

$\mathscr{L}=\left(\frac{g_{2}}{\sqrt{2}} W_{\mu}^{+} \bar{U}_{\mathrm{L}} \gamma^{\mu} \frac{m_{j}^{\prime *}}{M_{\mathrm{Q}}} C_{j i} d_{\mathrm{L} i}\right.$

$$
\left.+\frac{g_{2}}{2 c_{\mathrm{W}}} Z_{\mu} \bar{U}_{\mathrm{L}} \gamma^{\mu} \frac{m_{i}^{\prime *}}{M_{\mathrm{Q}}} u_{\mathrm{L} i}-H \bar{U}_{\mathrm{R}} \frac{m_{i}^{\prime *}}{v} u_{\mathrm{L} i}\right)+ \text { h.c. }
$$

$\mathrm{E}$

$$
\begin{aligned}
\mathscr{L}= & \left(\frac{g_{2}}{\sqrt{2}} W_{\mu}^{+} \bar{\nu}_{\mathrm{L} i} \gamma^{\mu} \frac{m_{i}^{\prime}}{M_{\mathrm{L}}} E_{\mathrm{L}}\right. \\
& \left.-\frac{g_{2}}{2 c_{\mathrm{W}}} Z_{\mu} \bar{e}_{\mathrm{L} i} \gamma^{\mu} \frac{m_{i}^{\prime}}{M_{\mathrm{L}}} E_{\mathrm{L}}-H \bar{e}_{\mathrm{L} i} \frac{m_{i}^{\prime}}{v} E_{\mathrm{R}}\right)+ \text { h.c. }
\end{aligned}
$$

$\left(\begin{array}{l}\mathrm{N} \\ \mathrm{E}\end{array}\right)$

$$
\begin{aligned}
\mathscr{L}= & \left(-\frac{g_{2}}{\sqrt{2}} W_{\mu}^{+} \bar{N}_{\mathrm{R}} \gamma^{\mu} \frac{m_{j}}{M_{\mathrm{L}}} e_{\mathrm{R} j}\right. \\
& \left.+\frac{g_{2}}{2 c_{\mathrm{w}}} Z_{\mu} \bar{E}_{\mathrm{R}} \gamma^{\mu} \frac{m_{j}}{M_{\mathrm{L}}} e_{\mathrm{R} j}-H \bar{E}_{\mathrm{L}} \frac{m_{j}}{v} e_{\mathrm{R} j}\right)+ \text { h.c. }
\end{aligned}
$$


where the $3 \times 3$ matrix $\Delta$ includes the standard up quark masses, the $C$ matrix and a new mixing matrix $C^{\prime}$, which is a priori partially observable. One could eventually measure $m_{i}$ and $C_{i j}^{\prime} m_{i}^{*} \equiv \tilde{m}_{i}^{*}$, which are the effective mixing parameters. Contributions of order $m_{i} m_{j} / M\left(m_{i} m_{j} / M^{2}\right)$ to light fermion masses (CabibboKobayashi-Maskawa matrices) are negligible in our analysis. The absence of $\nu_{R}$ in the minimal standard model simplifies the corresponding leptonic lagrangian terms in table 1. An important observation is that the dominant decay terms are all proportional.

Thus a new vector-like fermion $\mathrm{F}$ decays into $\mathrm{W}^{ \pm} \mathrm{f}, \mathrm{Zf}$ and $\mathrm{Hf}$ (where $\mathrm{f}$ stands for a standard fermion) with definite ratios, except for kinematical factors. The rates of this novel source of boson production are similar for $\mathrm{W}^{ \pm}, \mathrm{Z}$ and $\mathrm{H}$. This distinguishes vector-like fermions, because if copiously produced at hadron colliders they transform the latter into a small Higgs factory. The general conclusions for these minimal extensions of the standard model will survive obvious generalizations as we argue in sect. 4. Armed with table 1 , we can now discuss production rates (we concentrate on hadron colliders) and branching ratios. Note that new fermions are mainly pair-produced because their mixing reduces associated production with standard fermions.

\subsection{NEW VECTOR-LIKE QUARKS}

The most efficient way of pair-producing vector-like quarks, $Q \bar{Q}$, is through strong interactions. The tree level cross section $\mathrm{d} \sigma / \mathrm{d} \hat{t}$ for initial gg $(\mathrm{q} \bar{q})$ partons [16] is collected in ref. [17], eq. (5.17) [eq. (5.18)] and its erratum, in standard notation. (Weak production from longitudinal $\mathrm{W}$ and $\mathrm{Z}$ fusion [18] is negligible for heavy vector-like fermions which get their masses from explicit mass terms, and not from the standard Yukawa couplings. Then scalar couplings result from mixing and are suppressed.) The total cross section reduces to [17]

$$
\begin{aligned}
\sigma(a b \rightarrow \mathrm{Q} \overrightarrow{\mathrm{Q} X})= & \int_{2 M_{\mathrm{Q}}}^{\sqrt{s}} \mathrm{~d} M \int_{\ln (M / \sqrt{s})}^{\ln (\sqrt{s} / M)} \mathrm{d} y \int_{-z_{0}}^{z_{0}} \mathrm{~d} z \frac{2}{M} x_{a} x_{b} \\
\times & \left\{\sum_{\text {flavours }}\left[f_{\mathrm{q}}^{(a)}\left(x_{a}, M^{2}\right) f_{\overline{\mathrm{q}}}^{(b)}\left(x_{b}, M^{2}\right)+f_{\overline{\mathrm{q}}}^{(a)}\left(x_{a}, M^{2}\right) f_{\mathrm{q}}^{(b)}\left(x_{b}, M^{2}\right)\right]\right. \\
& \times \frac{\mathrm{d} \sigma}{\mathrm{d} z}(\mathrm{q} \overline{\mathrm{q}} \rightarrow \mathrm{QQ}) \\
& \left.+f_{\mathrm{g}}^{(a)}\left(x_{a}, M^{2}\right) f_{\mathrm{g}}^{(b)}\left(x_{b}, M^{2}\right) \frac{\mathrm{d} \sigma}{\mathrm{d} z}(\mathrm{gg} \rightarrow \mathrm{Q} \overline{\mathrm{Q}})\right\} .
\end{aligned}
$$

where $M$ is the $\mathrm{Q} \overline{\mathrm{Q}}$ invariant mass, $y$ the rapidity of the colliding partons 
TABLE 2

Production cross sections for heavy quarks at present and future colliders. The expected number of events is also given for the corresponding nominal luminosities

\begin{tabular}{|c|c|c|c|c|}
\hline Collider $(\sqrt{s} \mathrm{TeV})$ & $\begin{array}{c}\text { Vector-like } \\
\text { quark mass } \\
(\mathrm{GeV})\end{array}$ & $\begin{array}{c}\mathrm{QQ} \text { cross } \\
\text { section } \\
(\mathrm{pb})\end{array}$ & $\begin{array}{c}\int \mathscr{L} \mathrm{d} t \\
\left(\mathrm{pb}^{-1} \mathrm{y}^{1}\right)\end{array}$ & $\begin{array}{c}\text { Number of } \\
\text { events } \\
\text { per year }\end{array}$ \\
\hline Tevatron $\bar{p} \bar{p}(1.8)$ & 150 & 8 & 10 & 80 \\
\hline UNK pp $(6)$ & 300 & 7 & 100 & 700 \\
\hline LHC $p p(17)$ & 700 & 1.1 & $10^{4}$ & $11 \times 10^{3}$ \\
\hline $\mathrm{SSC} p(40)$ & 700 & 14 & $10^{4}$ & $140 \times 10^{3}$ \\
\hline
\end{tabular}

$\left(x_{a, b}=(M / \sqrt{s}) \mathrm{e}^{ \pm y}\right.$ and $z=\cos \theta^{*}, \theta^{*}$ the scattering angle of $a \mathrm{Q}$ in the center of mass of the parton system QQ $\bar{Q}$. Furthermore, $z_{0}=\min \left[\beta_{\mathrm{Q}}^{-1} \tanh (\ln (\sqrt{s} / M)-|y|, 1]\right.$, $\beta_{\mathrm{Q}}=\left(1-4 M_{\mathrm{Q}}^{2} / M^{2}\right), \quad f$ are the parton distribution functions and $\mathrm{d} \sigma / \mathrm{d} z$ $=\frac{1}{2} M^{2} \beta_{\mathrm{Q}} \mathrm{d} \sigma / \mathrm{d} \hat{t}$. We collect in table 2 the corresponding rates for present and future colliders and for illustrative vector-like quark masses $M_{\mathrm{Q}}$. (We do not consider the CERN collider because for $M_{\mathrm{Q}}>M_{\mathrm{W}}$ the cross sections are too small [19].) We use EHLQ structure functions, set 1 [17], through the paper, with $Q^{2}=4 M_{\mathrm{Q}}^{2}$ in this case. Other structure functions $[20,21]$ give similar results. Higher order (strong) corrections are known [22] but we neglect them within our approximations. Numerical results must be understood as estimates because we do not include fragmentation, detector simulation, etc.... The production cross sections are those of a sequential quark because strong interactions are blind to the electroweak quantum numbers. This makes the vector-like quark branching ratios essential in order to distinguish a vector-like quark from a sequential one.

The branching ratios for the decay of the new quarks are immediately calculated using the corresponding decay rates [13]

$$
\begin{aligned}
\Gamma\left(\mathrm{Q} \rightarrow \mathrm{Wq}_{i}\right) & =\frac{\alpha_{2}}{16} \frac{\left.\mid m_{i}^{(}\right)\left.\right|^{2}}{M_{\mathrm{Q}}^{2}} \frac{\lambda^{1 / 2}\left(M_{\mathrm{Q}}^{2}, M_{\mathrm{W}}^{2}, m_{\mathrm{q}_{i}}^{2}\right)}{M_{\mathrm{Q}}^{3}}\left[M_{\mathrm{Q}}^{2}-2 M_{\mathrm{W}}^{2}+m_{\mathrm{q}_{i}}^{2}+\frac{\left(M_{\mathrm{Q}}^{2}-m_{\mathrm{q}_{i}}^{2}\right)^{2}}{M_{\mathrm{W}}^{2}}\right] \\
\Gamma\left(\mathrm{Q} \rightarrow \mathrm{Hq}_{i}\right) & =\frac{\alpha_{2}}{32} \frac{\left.\mid m_{i}^{(}\right)\left.\right|^{2}}{M_{\mathrm{Q}}^{2}} \frac{\lambda^{1 / 2}\left(M_{\mathrm{Q}}^{2}, M_{\mathrm{H}}^{2}, m_{\mathrm{q}_{i}}^{2}\right)}{M_{\mathrm{Q}} M_{\mathrm{W}}^{2}}\left(M_{\mathrm{Q}}^{2}-M_{\mathrm{H}}^{2}+m_{\mathrm{q}_{i}}^{2}\right)
\end{aligned}
$$

with $\lambda(a, b, c)=a^{4}+b^{4}+c^{4}-2 a^{2} b^{2}-2 a^{2} c^{2}-2 b^{2} c^{2} . \quad \Gamma\left(\mathrm{Q} \rightarrow \mathrm{Zq}_{i}\right)$ is obtained from $\Gamma\left(\mathrm{Q} \rightarrow \mathrm{Wq}_{i}\right)$ by replacing $M_{\mathrm{W}}$ by $M_{\mathrm{Z}}$ and dividing the whole expression by $2 \cos ^{2} \theta_{\mathrm{w}}$. We have taken into account the mass of the final quark $m_{\mathrm{q}}$ (we use $m_{1}=100 \mathrm{GeV}$ ) but neglected the $C^{\left({ }^{\prime}\right)}$ mixing. Branching ratios, as decay rates, depend on the masses of the particles involved and on the mixing parameters $m_{i}^{()^{\prime}}$. However, neglecting standard fermion masses total branching ratios into $\mathrm{W}^{ \pm}, \mathrm{Z}$ 
TABLE 3

Decay branching ratios for a vector-like heavy quark ${ }^{\mathbf{a}}$

\begin{tabular}{|c|c|c|c|c|c|c|}
\hline & \multicolumn{3}{|c|}{ D quark type } & \multicolumn{3}{|c|}{ U quark type } \\
\hline & $\mathrm{Q} \rightarrow \mathrm{Wq}$ & $\mathrm{Q} \rightarrow \mathrm{Zq}$ & $\mathrm{Q} \rightarrow \mathrm{Hq}$ & $\mathrm{Q} \rightarrow \overline{\mathrm{Wq}}$ & $\bar{Q} \rightarrow \mathrm{Zq}$ & $\mathrm{Q} \rightarrow \mathrm{Hq}$ \\
\hline \multicolumn{7}{|l|}{$M_{\mathrm{Q}}=150 \mathrm{GeV}$} \\
\hline \multirow[t]{3}{*}{$M_{\mathrm{H}}=60 \mathrm{GeV}$} & 0.0 & 0.50 & 0.50 & 1.0 & 0.0 & 0.0 \\
\hline & 0.42 & 0.29 & 0.29 & 0.62 & 0.19 & 0.19 \\
\hline & 0.53 & 0.23 & 0.24 & 0.53 & 0.23 & 0.24 \\
\hline \multirow[t]{3}{*}{$M_{\mathrm{H}}=92 \mathrm{GeV}$} & 0.0 & 0.64 & 0.36 & 1.0 & 0.0 & 0.0 \\
\hline & 0.49 & 0.33 & 0.18 & 0.68 & 0.21 & 0.11 \\
\hline & 0.59 & 0.26 & 0.15 & 0.59 & 0.26 & 0.15 \\
\hline \multicolumn{7}{|l|}{$M_{\mathrm{Q}}=300 \mathrm{GeV}$} \\
\hline \multirow{3}{*}{$M_{\mathrm{H}}=92 \mathrm{GeV}$} & 0.43 & 0.31 & 0.26 & 0.57 & 0.20 & 0.23 \\
\hline & 0.49 & 0.28 & 0.23 & 0.53 & 0.25 & 0.22 \\
\hline & 0.52 & 0.26 & 0.22 & 0.52 & 0.26 & 0.22 \\
\hline \multirow[t]{3}{*}{$M_{\mathrm{H}}=150 \mathrm{GeV}$} & 0.47 & 0.34 & 0.19 & 0.63 & 0.22 & 0.15 \\
\hline & 0.53 & 0.30 & 0.17 & 0.58 & 0.26 & 0.16 \\
\hline & 0.56 & 0.28 & 0.16 & 0.56 & 0.28 & 0.16 \\
\hline \multicolumn{7}{|l|}{$M_{\mathrm{Q}}=700 \mathrm{GeV}$} \\
\hline \multirow[t]{3}{*}{$M_{\mathrm{H}}=150 \mathrm{GeV}$} & 0.49 & 0.27 & 0.24 & 0.52 & 0.25 & 0.23 \\
\hline & 0.50 & 0.27 & 0.23 & 0.51 & 0.26 & 0.23 \\
\hline & 0.51 & 0.26 & 0.23 & 0.51 & 0.26 & 0.23 \\
\hline \multirow{3}{*}{$M_{\mathrm{H}}=300 \mathrm{GeV}$} & 0.52 & 0.29 & 0.19 & 0.55 & 0.27 & 0.18 \\
\hline & 0.54 & 0.28 & 0.18 & 0.54 & 0.28 & 0.18 \\
\hline & 0.54 & 0.28 & 0.18 & 0.54 & 0.28 & 0.18 \\
\hline
\end{tabular}

${ }^{a}$ For each boson we sum all the standard quark contributions. We take $m_{\mathrm{t}}=100 \mathrm{GeV}$ and different vector-like quark and Higgs masses. For each choice we display three cases: $M_{\mathrm{Q}} \gg\left|m_{3}^{\left.()^{\prime}\right)}\right| \gg\left|m_{1.2}^{\left(\gamma_{2}\right.}\right|$, first row: $M_{\mathrm{Q}} \gg\left|m_{1}^{(\prime)}\right|=\left|m_{2}^{(\prime)}\right|=\left|m_{3}^{\left({ }^{\prime}\right)}\right|$, second row; $M_{\mathrm{Q}} \gg\left|m_{1,2}^{(\prime)}\right| \gg\left|m_{3}^{(\prime)}\right|$, third row.

and/or $\mathrm{H}$ are independent of the mixing parameters. (That is, the factors $\left|m_{i}^{\left({ }^{\prime}\right)}\right|^{2} / M_{\mathrm{Q}}^{2}$ are common to all the decays. Sometimes those ratios are written as additional mixing angles [9].) This may not be a good approximation for the top quark mass, $m_{t}$. At any rate, according to table 1 , a large $m_{t}$ would eventually translate into a suppression of the Higgs decay rate for a new $U$ quark singlet only for the case $m_{3}^{\prime} \gg m_{1,2}^{\prime}$. For a new D quark singlet such a heavy top results in an enhancement of the Higgs branching ratio, whereas for a new $\left(\begin{array}{l}U \\ D\end{array}\right)$ quark doublet the suppression or the enhancement depend on the particular signal. We postpone any further discussion until sect. 3 . In table 3 we give for illustration the branching ratios for different mass values and mixing angles.

\subsection{NEW VECTOR-LIKE LEPTONS}

As in the case of vector-like quarks, vector-like leptons couple little to the Higgs and to longitudinal electroweak gauge bosons. (This makes inefficient the 
gluon-gluon and electroweak gauge boson fusion mechanisms [18,23].) Then these new leptons are mainly produced by the standard Drell-Yan mechanism. The cross section for a charged lepton can be written

$$
\begin{array}{r}
\sigma(a b \rightarrow \mathrm{L} \overline{\mathrm{L} X})=\int_{2 M_{\mathrm{L}}}^{\sqrt{s}} \mathrm{~d} M \int_{\ln (M / \sqrt{s})}^{\ln (\sqrt{s} / M)} \mathrm{d} y \int_{-z_{0}}^{z_{0}} \mathrm{~d} z \frac{2}{M} x_{a} x_{b} \\
\quad \times\left\{\sum _ { \text { flavours } } \frac { 1 } { 3 } \left[f_{\mathrm{q}}^{(a)}\left(x_{a}, M^{2}\right) f_{\overline{\mathrm{q}}}^{(b)}\left(x_{b}, M^{2}\right)\right.\right. \\
\left.\left.+f_{\overline{\mathrm{q}}}^{(a)}\left(x_{a}, M^{2}\right) f_{\mathrm{q}}^{(b)}\left(x_{b}, M^{2}\right)\right] \frac{\mathrm{d} \sigma_{\mathrm{q}}}{\mathrm{d} z}\right\},
\end{array}
$$

where $z_{0}=\min \left[\beta_{\mathrm{L}}^{-1} \tanh (\ln (\sqrt{s} / M)-|y|), 1\right], \beta_{\mathrm{L}}=\left(1-4 M_{\mathrm{L}}^{2} / M^{2}\right)$ and (in standard notation) $[13,24]$

$$
\begin{aligned}
\frac{\mathrm{d} \sigma_{\mathrm{q}}}{\mathrm{d} z}=\frac{\pi \alpha^{2}}{M^{2}} \frac{\beta_{\mathrm{L}}}{2}\{ & e_{\mathrm{L}}^{2} e_{\mathrm{q}}^{2}\left(2-\beta_{\mathrm{L}}^{2}+\beta_{\mathrm{L}}^{2} z^{2}\right) \\
& +2 e_{\mathrm{L}} e_{\mathrm{q}} \xi_{1}\left[v_{\mathrm{L}} v_{\mathrm{q}}\left(2-\beta_{\mathrm{L}}^{2}+\beta_{\mathrm{L}}^{2} z^{2}\right)+a_{\mathrm{L}} a_{\mathrm{q}} 2 \beta_{\mathrm{L}} z\right] \\
+ & \xi_{2}\left[v_{\mathrm{L}}^{2}\left(v_{\mathrm{q}}^{2}+a_{\mathrm{q}}^{2}\right)\left(2-\beta_{\mathrm{L}}^{2}+\beta_{\mathrm{L}}^{2} z^{2}\right)\right. \\
& \left.\left.+\beta_{\mathrm{L}}^{2} a_{\mathrm{L}}^{2}\left(v_{\mathrm{q}}^{2}+a_{\mathrm{q}}^{2}\right)\left(1+z^{2}\right)+v_{\mathrm{L}} a_{\mathrm{L}} v_{\mathrm{q}} a_{\mathrm{q}} 8 \beta_{\mathrm{L}} z\right]\right\},
\end{aligned}
$$

where

$$
\begin{aligned}
& \xi_{1}=\frac{1}{16 s_{\mathrm{W}}^{2} c_{\mathrm{W}}^{2}} \frac{M^{2}\left(M^{2}-M_{\mathrm{Z}}^{2}\right)}{\left(M^{2}-M_{\mathrm{Z}}^{2}\right)^{2}+\Gamma_{\mathrm{Z}}^{2} M_{\mathrm{Z}}^{2}}, \\
& \xi_{2}=\frac{1}{256 s_{\mathrm{W}}^{2} c_{\mathrm{W}}^{2}} \frac{M^{4}}{\left(M^{2}-M_{\mathrm{Z}}^{2}\right)^{2}+\Gamma_{\mathrm{Z}}^{2} M_{\mathrm{Z}}^{2}}
\end{aligned}
$$

and the different charges are given in table 4. (In ref. [20] there is an extra factor $\left(3-\beta_{\mathrm{L}}\right) / 2$ in $\mathrm{d} \sigma_{\mathrm{q}} / \mathrm{d} z$ which we do not find.) The difference with the standard model case is the vector-like character (couplings) of the new leptons (see tables 1 and 4). For a new vector-like lepton $\operatorname{doublet}\left(\begin{array}{l}\mathrm{N} \\ \mathrm{E}\end{array}\right)$ the same equations apply for $\mathrm{NN}$ and $\bar{E} \bar{E}$ production, but with the corresponding changes in table 4. For NE production the $\mathrm{W}$ interchange gives a $\mathrm{do}_{\mathrm{ud}} / \mathrm{d} z$ formally equal to eq. (2.6) but without the photon interchange (terms proportional to $e_{\mathrm{L}} e_{\mathrm{q}}$ ) and with $M_{Z}, \Gamma_{\mathrm{Z}}$ replaced by $M_{\mathrm{W}}, \Gamma_{\mathrm{W}}$. The corresponding charges $Q_{i}^{\mathrm{ud} . \mathrm{NE}}$ are also given in table 4 . 
TABLE 4

Electric charge $e_{f}$, and vector $v_{f}$ and axial $a_{f}$ couplings for standard quarks and for vector-like leptons

\begin{tabular}{lrcr}
\hline Fermion & $e_{f}$ & $v_{f}$ & $a_{f}$ \\
\hline u-quark type & $\frac{2}{3}$ & $1-\frac{8}{3} s_{\mathrm{w}}^{2}$ & 1 \\
d-quark type & $-\frac{1}{3}$ & $-1+\frac{4}{3} s_{\mathrm{W}}^{2}$ & -1 \\
E singlet & -1 & $4 s_{\mathrm{W}}^{2}$ & 0 \\
N doublet & 0 & 2 & 0 \\
E doublet & -1 & $-2+4 s_{\mathrm{W}}^{2}$ & 0 \\
ud charge & - & $\sqrt{2} c_{\mathrm{w}}$ & $\sqrt{2} c_{\mathrm{W}}$ \\
N E charge & - & $2 \sqrt{2} c_{\mathrm{W}}$ & 0 \\
\hline
\end{tabular}

(Eq. (2.5) must be trivially modified to allow for the ud annihilation.) Table 5 collects the total cross sections [eq. (2.5)] for different lepton masses and hadron colliders, for illustration. Comparing tables 2 and 5 , it is apparent that leptons are more difficult to produce than quarks at hadron colliders. However, signals are more striking and backgrounds less important for the same reason, as we discuss in subsect. 2.6. The decays of these new leptons are governed by the corresponding lagrangian terms in table 1 . In all cases total branching ratios into $\mathrm{W}^{ \pm}, \mathrm{Z}$ and $\mathrm{H}$ are independent of the mixing parameters $m_{i}^{(\prime)}$ because standard lepton masses are negligible! (There is the subtlety, however, of a possible large $\tau$ branching ratio $\left(m_{3}^{(\prime)} \gg m_{1,2}^{(\prime)}\right)$, in which case $\tau$ misidentification may become a problem.) Table 6 collects, for illustration, the branching ratios for different mass values (the decay rate expressions are analogous to those of vector-like quarks, eq. (2.4)). As can be observed, decay rates into Higgs are always large - given that $\mathrm{N}$ and $\mathrm{E}$ are almost degenerate in mass for the $\left(\begin{array}{l}\mathrm{N} \\ \mathrm{E}\end{array}\right)$ doublet, their production and signals must always be summed up! Let us now translate the total cross section numbers into more realistic numbers for signals and backgrounds.

TABLE 5

Production cross sections (in pb) for heavy vector-like leptons at present and future colliders

\begin{tabular}{|c|c|c|c|c|c|}
\hline \multirow[t]{2}{*}{ Collider } & \multirow[t]{2}{*}{$M_{\mathrm{L}}(\mathrm{GeV})$} & \multirow[t]{2}{*}{ E } & \multicolumn{3}{|c|}{$\left(\begin{array}{l}N \\
E\end{array}\right)$} \\
\hline & & & $\mathrm{NN}$ & $\mathrm{EE}$ & $\mathrm{NE}^{-}+\mathrm{NE}^{+}$ \\
\hline Tevatron & 100 & 0.1 & 0.3 & 0.3 & 0.6 \\
\hline UNK & 200 & 0.05 & 0.10 & 0.12 & 0.29 \\
\hline LHC & 500 & $4 \times 10^{-3}$ & $9 \times 10^{-3}$ & 0.01 & 0.03 \\
\hline SSC & 500 & 0.02 & 0.04 & 0.04 & 0.13 \\
\hline
\end{tabular}


Decay branching ratios for vector-like heavy leptons. For each boson we sum all the standard lepton contributions

\begin{tabular}{|c|c|c|c|c|c|c|c|c|c|}
\hline & \multicolumn{3}{|c|}{$\mathrm{E}$} & \multicolumn{6}{|c|}{$\left(\begin{array}{l}N \\
E\end{array}\right)$} \\
\hline & & & & \multicolumn{3}{|c|}{$\mathrm{N}$} & \multicolumn{3}{|c|}{$\mathrm{E}$} \\
\hline & $\mathrm{L} \rightarrow \mathrm{W} \nu$ & $\mathrm{L} \rightarrow \mathrm{Z} \ell$ & $\overline{\mathrm{L} \rightarrow \mathrm{H} \ell}$ & $\mathrm{L} \rightarrow \mathrm{W} \ell$ & $\mathrm{L} \rightarrow \mathrm{Z} \nu$ & $\overline{\mathrm{L} \rightarrow \mathrm{H} v}$ & $\mathrm{~L} \rightarrow \mathrm{W} v$ & $\mathrm{~L} \rightarrow \mathrm{Z} \ell$ & $\overline{\mathrm{L} \rightarrow \mathrm{H} \ell}$ \\
\hline \multicolumn{10}{|l|}{$M_{\mathrm{L}}=100 \mathrm{GeV}$} \\
\hline$M_{\mathrm{H}}=60 \mathrm{GeV}$ & 0.51 & 0.07 & 0.42 & 1.0 & 0.0 & 0.0 & 0.0 & 0.14 & 0.86 \\
\hline$M_{\mathbf{H}}=92 \mathrm{GeV}$ & 0.85 & 0.11 & 0.04 & 1.0 & 0.0 & 0.0 & 0.0 & 0.74 & 0.26 \\
\hline \multicolumn{10}{|l|}{$M_{\mathrm{L}}=200 \mathrm{GeV}$} \\
\hline$M_{\mathrm{H}}=92 \mathrm{GeV}$ & 0.55 & 0.27 & 0.18 & 1.0 & 0.0 & 0.0 & 0.0 & 0.59 & 0.41 \\
\hline$M_{\mathbf{H}}=150 \mathrm{GeV}$ & 0.63 & 0.31 & 0.06 & 1.0 & 0.0 & 0.0 & 0.0 & 0.83 & 0.17 \\
\hline \multicolumn{10}{|l|}{$M_{\mathrm{L}}=500 \mathrm{GeV}$} \\
\hline$M_{\mathrm{H}}=150 \mathrm{GeV}$ & 0.52 & 0.27 & 0.21 & 1.0 & 0.0 & 0.0 & 0.0 & 0.55 & 0.45 \\
\hline$M_{\mathrm{H}}=300 \mathrm{GeV}$ & 0.58 & 0.30 & 0.12 & 1.0 & 0.0 & 0.0 & 0.0 & 0.72 & 0.28 \\
\hline
\end{tabular}

\section{Signatures and backgrounds}

Heavy fermion $F \bar{F}$ signals (and backgrounds) at hadron colliders can be classified by the number of lepton pairs (we include the neutrino missing $p_{\mathrm{t}}$ for this counting) and the number of large $p_{\mathrm{t}}$ jets. In fact, vector-like fermions are pair produced and tend to decay very fast into a standard fermion and an electroweak boson, the latter decaying into two additional fermions (this if $M_{\mathrm{H}}<2 M_{\mathrm{W}}$, in the Higgs boson case). Therefore, their signals are basically 6 leptons, 4 leptons +2 jets, 2 leptons +4 jets and 6 jets. The backgrounds one should consider are standard model processes leading to the corresponding six fermion states. Their matrix elements are not known at the moment, so one has to design a strategy in order to get a rough estimate of their contributions.

Our study is done at the parton level (without parton fragmentation, detector simulation, ...) where a quark or a gluon in a final state is considered a hadronic jet, and cuts are directly applied to leptons and jets. We essentially apply two cuts to signals and backgrounds. Final fully detectable fermions (charged leptons and jets) are required to lie in a given interval of pseudorapidity, $|\eta|<\eta^{\max }$, (this means keeping the central events), and, in addition, to have a minimum transverse momentum, $p_{\mathrm{t}}>p_{\mathrm{t}}^{\mathrm{min}}$, (including in this case neutrinos). Knowing that signals can be considered as isotropically produced (in first approximation) and that the $p_{t}$ of the different final products tends to be large for large $F \bar{F}$ invariant masses, whilst backgrounds prefer the forward-backward direction and low $p_{\mathrm{t}}$ values, our cuts will improve the signal/background ratios. We choose typical $p_{\mathrm{t}}^{\min }$ values for every collider we consider and a common $\eta^{\max }=4$, for illustration. 
Our final goal is to determine the feasibility of detecting a (intermediate) Higgs boson, once a $\bar{F} \bar{F}$ has been produced. We concentrated on six final fermion signals, which are the relevant ones for observing a new fermion. In these samples there will be a sizable percentage of events with a Higgs (for a vector-like fermion) if $\mathrm{H} \rightarrow \mathrm{q} \overline{\mathrm{q}}$. For a heavy Higgs $\left(M_{\mathrm{H}}>2 M_{\mathrm{W}}\right)$ which will decay mainly into WW, ZZ, one should look to the 8 (10) final fermion signals for observing the Higgs, once the existence of the heavy vector-like fermion is established by looking at the six fermion modes.

For both quark and leptons, the important signals will be $(\ell \bar{\ell}, \ell \nu)+$ jijj. For a quark one selects those events with the lepton pair reconstructing a $W$ or a $Z$ boson. For a lepton one does the opposite: one cuts on the lepton pair invariant mass to make sure it has not originated from a W or $\mathrm{Z}$ decay, $M_{\ell \dot{\ell}_{,}, \nu} \gg M_{\mathrm{Z}}$. In such a reduced sample one looks for events with $M_{\left(\ell \bar{\ell}, \ell v \mathrm{j}_{1}\right.} \simeq M_{\mathrm{j}_{2} \mathrm{j}_{3} \mathrm{j}_{4}} \simeq M_{\mathrm{Q}_{-}}\left(M_{\ell \mathrm{j}_{1} \mathrm{j}_{2}} \simeq\right.$ $\left.M_{(\bar{\ell}, v) \mathrm{j}_{3} \mathrm{j}_{4}} \simeq M_{\mathrm{L}}\right)$ in order to isolate those events coming from $\mathrm{QQ}(\mathrm{L} \overline{\mathrm{L}})$ and to determine the heavy quark (lepton) mass $M_{\mathrm{Q}}\left(M_{\mathrm{L}}\right)$. In the lepton case the two jets accompanying the leptons often reconstruct a $\mathrm{W}$ or $\approx \mathrm{Z}$. Then comparing the charged $(\ell \nu \mathrm{jjjj})$ and neutral $(\ell \bar{\ell} \mathrm{jjjj})$ signals, the vector-like or sequential character of the new fermion can be decided. Once $M_{\mathrm{F}}$ is known one comes back to the initial total $F \bar{F}$ sample (that is to say, one associates three fermions with $F$ and the other three fermions with $\overline{\mathbf{F}}$ ). An analysis of the two-jet invariant mass should then signal the presence of the Higgs channel (together with the $\mathrm{W}$ and $\mathrm{Z}$ channels) and allow for the determination of the Higgs mass.

Signals are estimated using the expressions given in sect. 2 and assuming isotropic, on-shell decays of vector-like fermions and subsequent bosons. (Results are compatible within our approximations with a proper treatment of the polarization amplitudes [25].)

\subsection{VECTOR-LIKE QUARKS}

The signatures from $Q \bar{Q}$ production have 6 jets, 2 leptons +4 jets and 4 leptons plus 2 jets. In table $7 \mathrm{a}$ we give the fraction of $\mathrm{Q} \overline{\mathrm{Q}}$ decays into the channels with at most one neutrino (in parentheses there is for each case the fraction of events which are Higgs mediated if $\mathrm{H} \rightarrow \mathrm{q} \overline{\mathrm{q}}$ ). $\ell$ stands for $\mathrm{e}^{+}$and $\mu^{ \pm}$and $\nu$ for their neutrinos. In table $7 \mathrm{~b}$ we quote the cross sections (in the absence of any cut) for different colliders and for the $Q$ masses indicated for the signals in table 7a involving leptons.

The results of table $7 \mathrm{a}$ were obtained using $m_{\mathrm{t}}=100 \mathrm{GeV}$ and the standard model decay rates for $\mathrm{W}$ and $\mathrm{Z}$, whereas the Higgs was supposed to decay into quarks only. For calculating the fractions in table 7 we approximated all different cases in table 3 to a common average ratio: $\mathrm{W} / \mathrm{Z} / \mathrm{H}=2 / 1 / 1$, resulting in the branching ratios

$$
\mathrm{WW} / \mathrm{ZZ} / \mathrm{HH} / \mathrm{WZ} / \mathrm{WH} / \mathrm{ZH}=4 / 1 / 1 / 4 / 4 / 2 .
$$

In the absence of final flavour identification there is no difference between $\mathrm{U}$ and $\mathrm{D}$ 
TABLE $7 \mathrm{a}$

Fraction of (vector-like) $Q \bar{Q}$ decays into final states with at most one neutrino. $\ell$ stands for e and $\mu$. We take an average for the branching ratio into $W, Z$ and $H$ (see text) and assume that $H$ always decays into $\mathrm{q} \overline{\mathrm{q}}$. For each mode we give in parentheses the fraction of events with at least one Higgs

\begin{tabular}{ccc}
\hline Signal & $\begin{array}{c}\text { Decay mode } \\
\text { fraction }\end{array}$ & $\mathrm{D}=\mathrm{U}=\left(\begin{array}{c}\mathrm{U} \\
\mathrm{D}\end{array}\right)$ \\
$\begin{array}{c}\text { (Fraction of events } \\
\text { with a Higgs) }\end{array}$ \\
$\mathrm{q} \overline{\mathrm{q}} \ell \bar{\ell} \ell \bar{\ell}$ & $\begin{array}{c}3 \times 10^{-4} \\
\mathrm{q} \overline{\mathrm{q}} \ell \bar{\ell} \ell \nu\end{array}$ & $(-)$ \\
$\mathrm{q} \overline{\mathrm{q}} \mathrm{q} \overline{\mathrm{q}} \ell \bar{\ell}$ & $4 \times 10^{-3}$ & $(-)$ \\
$\mathrm{q} \overline{\mathrm{q}} \mathrm{q} \overline{\mathrm{q}} \ell \nu$ & 0.03 & $(0.33)$ \\
$\mathrm{q} \overline{\mathrm{q}} \mathrm{q} \overline{\mathrm{q}} \mathrm{q} \overline{\mathrm{q}}$ & 0.17 & $(0.55)$ \\
\hline
\end{tabular}

quark singlets, whereas the difference with the $\left(\begin{array}{l}U \\ D\end{array}\right)$ doublet is simply that in this case both $\mathrm{U}$ and $\mathrm{D}$ contributions have to be summed up, due to the assumed degeneracy of their masses, resulting in cross sections a factor of two larger than those of the singlet cases (table 7b). This may not be enough to distinguish between singlet and doublet vector-like quarks in hadron colliders. The best way to distinguish between them and a sequential quark is provided by the ratio

$$
\ell \bar{\ell} \mathrm{jj \textrm {jj }} / \ell \nu \mathrm{j \textrm {jjj }}-\frac{1}{6}(0)
$$

for a vector-like (sequential) quark.

TABLE $7 b$

Total cross sections (in pb) for the signals with at least one lepton, and for different colliders and illustrative vector-like quark masses

\begin{tabular}{|c|c|c|c|c|}
\hline & $\begin{array}{c}\mathrm{q} \overline{\mathrm{q}} \ell \bar{\ell} \ell \bar{\ell} \\
\mathrm{D}=\mathrm{U}=\frac{1}{2}\left(\begin{array}{l}\mathrm{U} \\
\mathrm{D}\end{array}\right)\end{array}$ & $\begin{array}{c}\mathrm{q} \overline{\mathrm{q}} \ell \bar{\ell} \ell \nu \\
\mathrm{D}=\mathrm{U}=\frac{1}{2}\left(\begin{array}{l}\mathrm{U} \\
\mathrm{D}\end{array}\right)\end{array}$ & $\begin{array}{c}\mathrm{q} \overline{\mathrm{q}} \mathrm{q} \overline{\mathrm{q}} \ell \bar{\ell} \\
\mathrm{D}=\mathrm{U}=\frac{1}{2}\left(\begin{array}{l}\mathrm{U} \\
\mathrm{D}\end{array}\right)\end{array}$ & $\begin{array}{c}\mathrm{q} \overline{\mathrm{q}} \mathrm{q} \overline{\mathrm{q}} \ell \nu \\
\mathrm{D}=\mathbf{U}=\frac{1}{2}\left(\begin{array}{l}\mathrm{U} \\
\mathrm{D}\end{array}\right)\end{array}$ \\
\hline $\begin{array}{c}\text { Tevatron } \\
M_{\mathrm{Q}}=150 \mathrm{GeV}\end{array}$ & $2 \times 10^{-3}$ & 0.03 & 0.2 & 1.3 \\
\hline $\begin{array}{c}\mathrm{UNK} \\
M_{\mathrm{Q}}=300 \mathrm{GcV}\end{array}$ & $2 \times 10^{-3}$ & 0.03 & 0.2 & 1.2 \\
\hline $\begin{array}{c}\text { LHC } \\
M_{\mathrm{Q}}=700 \mathrm{GeV}\end{array}$ & $3 \times 10^{-4}$ & $4 \times 10^{-3}$ & 0.03 & 0.2 \\
\hline $\begin{array}{c}\text { SSC } \\
M_{\mathrm{Q}}=700 \mathrm{GeV}\end{array}$ & $4 \times 10^{-3}$ & 0.05 & 0.4 & 2.4 \\
\hline
\end{tabular}


From table $7 \mathrm{a}$ it can be inferred that the most likely final state is the 6 yet one, whereas the 4 lepton +2 jet final states have the smallest branching ratios, and the 2 lepton +4 jet events the intermediate ones. Let us turn now to the discussion of the backgrounds of the $\mathrm{Q} \overline{\mathrm{Q}}$ signals.

The 6 jet signal competes with a presumably huge QCD background, and with additional background from $\bar{t}$ production. Although the complete $2 \mathrm{~g} \rightarrow 6 \mathrm{~g}$ matrix elements are already available [26], we did not consider such a signal in our analysis of $Q \bar{Q}$ production. On the contrary, the 4 lepton channels are the cleanest signals from the experimental point of view, but at the price of having cross sections which are too small. We are left therefore with 2 lepton channels and striking signatures of two charged or one charged and missing transverse momentum plus 4 jets in the final state. From table $7 \mathrm{a}$ one realizes that the charged current channel is a factor - 6 times larger than the neutral one, so for illustration we concentrate our discussion of backgrounds on the former case. (Remember that the ratio $\ell \bar{\ell}$ jijj $/ \ell \nu \mathrm{jijj} \sim 1 / 6(0)$ [eq. (3.2)] is the signature for vector-like (sequential) quarks, implying that the study of both signals is necessary for a complete analysis.)

There are essentially three sources of $\ell v \mathrm{jjjj}$ backgrounds in the standard model:

$$
\begin{aligned}
& \text { (i) } \overline{\mathrm{t}} \rightarrow(\mathrm{Wq})(\mathrm{W} \overline{\mathrm{q}}) \rightarrow \ell \nu \mathrm{q} \overline{\mathrm{q}} \mathrm{q} \overline{\mathrm{q}} \\
& \text { (ii) } \mathrm{q} \overline{\mathrm{q}} \mathrm{q} \overline{\mathrm{q}}(\mathrm{W} \rightarrow \ell \nu) \\
& \text { (iii) } \mathrm{q} \overline{\mathrm{q}}(\mathrm{W} \rightarrow \ell \nu)(\mathrm{W}, \mathrm{Z} \rightarrow \mathrm{q} \overline{\mathrm{q}})
\end{aligned}
$$

The matrix elements of processes (ii) and (iii) are not available at the moment. The same analysis we use for vector-like quarks can be applied to process (i), where the only uncertain quantity is the top quark mass. For processes (ii) and (iii) we proceed as follows. We take the background, Wjjjj or WWjj (with the appropriate subse-

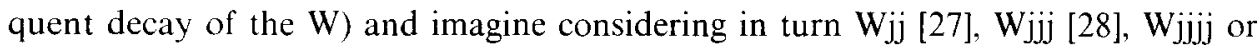
WW [29], WWj [30], WWjj and apply the same set of cuts (when possible) to all those processes with an increasing number of jets. Computing the cross sections for the first process in both cases (given a set of cuts), one can obtain a rough estimate of the other processes with additional jets multiplying the former results by a factor $\sim 0.2$ for each extra jet present in the final state. Such a strategy, although oversimplified, can be tested with the first step in the chains of additional jets and, at this stage, proves to give good estimations of the results $[28,30]$. This encourages us to go one step further and present (rough) results of two extra jets with respect to the initial, calculable processes. (Note that we cannot properly estimate the (essential) effect of $Q$ reconstruction, which makes the use of the correct matrix elements compulsory when they become available.)

The first process, $\mathrm{t}$ background, turns out to be indistinguishable (except for the total cross section) from the $\mathrm{QQ}$ signal in the $\ell \nu \mathrm{jjj}$ channel for $M_{\mathrm{Q}} \sim m_{1}$. One has 
TABLE 8

Expected number of events (for the luminosities in table 2) for $\mathrm{Q} \overline{\mathrm{Q}} \rightarrow \mathrm{WWjj} \rightarrow \ell \nu \mathrm{jjjj} ; \overline{\mathrm{tt}} \rightarrow \mathrm{WWjj} \rightarrow \ell \nu \mathrm{jjj}$ (where the top-type quark mass $m_{\mathrm{t}}=m_{\mathrm{Q}}$ ); $\mathrm{W} \mathrm{jj} \rightarrow \ell v \mathrm{jj}$, with the two QCD jets well separated in the lego plot $(\delta r>0.5)$ and "faking" a W $\left(M_{\mathrm{jj}} \in\left[M_{\mathrm{W}} \pm 10 \mathrm{GeV}\right]\right)$; and the WW $\rightarrow \ell \nu \mathrm{jj}$ continuum. We show the worst case with $m_{\mathfrak{t}}=M_{\mathrm{Q}}$; detection is simpler if the masses are different. All final fermions satisfy transverse momentum, $p_{t}$, and pseudorapidity (except for the neutrino), $\eta$. cuts. The $W_{j j}$ background is less of a problem for the SSC than for LHC because a larger $p_{\mathrm{t}}$ cut can be used. In parentheses we estimate the $\mathrm{Wjjj}_{\mathrm{jj}}(\mathrm{WWjj})$ backgrounds multiplying the $\mathrm{W}_{\mathrm{jj}}(\mathrm{WW})$ results by a reduction factor 0.2 for each additional jet. For the Tevatron, at the values used $\left(M_{\mathrm{Q}}=150 \mathrm{GeV} .10 \mathrm{pb}^{-1} / \mathrm{yr}\right)$ the number of events is too small for a signal to be detected, but if $M_{\mathrm{Q}}$ decreases a little or the luminosity increases a little, detection should be possible

\begin{tabular}{|c|c|c|c|c|}
\hline & $\begin{array}{c}\text { Tevatron } \\
{\left[M_{\mathrm{Q}}=150 \mathrm{GeV}\right]} \\
|\eta|<4 \\
p_{\mathrm{t}}>10 \mathrm{GeV}\end{array}$ & $\begin{array}{c}\text { UNK } \\
{\left[M_{\mathrm{Q}}=300 \mathrm{GeV}\right]} \\
|\eta|<4 \\
p_{\mathbf{t}}>10 \mathrm{GeV}\end{array}$ & $\begin{array}{c}\text { LHC } \\
{\left[M_{\mathrm{Q}}=700 \mathrm{GcV}\right]} \\
|\eta|<4 \\
p_{\mathrm{t}}>50 \mathrm{GeV}\end{array}$ & $\begin{array}{c}\mathrm{SSC} \\
{\left[M_{\mathrm{Q}}=700 \mathrm{GcV}\right]} \\
|\eta|<4 \\
p_{\mathrm{t}}>100 \mathrm{GeV}\end{array}$ \\
\hline \multicolumn{5}{|l|}{$\mathrm{Q} \overline{\mathrm{Q}} \rightarrow \mathrm{WWjj} \rightarrow \ell \nu \mathrm{jijj}$} \\
\hline $\mathrm{D}=\mathrm{U}=\frac{1}{2}\left(\begin{array}{l}\mathrm{U} \\
\mathrm{D}\end{array}\right)$ & 5 & 44 & 265 & 942 \\
\hline$\overline{\mathrm{t}} \rightarrow \mathrm{WW}_{\mathrm{jj}} \rightarrow \ell \nu \mathrm{jj \textrm {jj }}$ & 20 & 174 & 1059 & 3770 \\
\hline$W_{\mathrm{jj}} \rightarrow \ell v \mathrm{jj}$ & 174 & 19360 & 25800 & 1040 \\
\hline $\begin{array}{l}M_{\mathrm{jj}} \in\left[M_{\mathrm{W}} \pm 10 \mathrm{GeV}\right] \\
\delta r>0.5\end{array}$ & $(-7)$ & $(-774)$ & $(-1032)$ & $(-42)$ \\
\hline$W W \rightarrow \ell \nu \mathrm{jj}$ & $\begin{array}{c}13 \\
(-0.5)\end{array}$ & $\begin{array}{c}524 \\
(-21)\end{array}$ & $\begin{array}{c}1 \times 20 \\
(-73)\end{array}$ & $\begin{array}{r}590 \\
(-24)\end{array}$ \\
\hline
\end{tabular}

to rely on the $\ell \bar{\ell}$ jjjj signal to distinguish them: for $\mathrm{QQ}$ production the cross sections are smaller than for $\ell \nu \mathrm{jjjj}$, whereas for tit the $\ell \bar{\ell} \mathrm{jjjj}$ signal is absent (due to the absence of tree level flavour changing neutral currents for standard quarks). For $M_{\mathrm{Q}} \gg m_{\mathrm{t}}$ the top background in the charged channel could be reduced below the new signal.

In table 8 we give the estimated number of events (using the luminosities of table 2) for the $\ell \nu \mathrm{jjjj}$ signal (we took into account that only $44 \%$ of the signal comes from $\mathrm{W} \rightarrow \mathrm{q} \overline{\mathrm{q}}$, top, Wijjj and $\mathrm{WWjj}$ backgrounds. We demanded that the final fermions satisfy the pseudorapidity (except for the $\nu$ ) and transverse momentum cuts specified in table 8 . We also give the expected number of events for $\mathrm{Wjj}$ and $\mathrm{WW}$ processes. The $\mathrm{Wjj}$ sample was demanded to satisfy two additional cuts: $M_{\mathrm{ji}} \in\left[M_{\mathrm{W}}\right.$ $\pm 10 \mathrm{GeV}]$, in which two jets "fake" a W, and with both jets well separated in the lego plot, $\delta r=\sqrt{(\delta \phi)^{2}+(\delta \eta)^{2}}>0.5$, where $\delta \phi$ is the separation on the azimuthal angle in the transverse plane and $\delta \eta$ the corresponding separation in pseudorapidity. We find, with our set of cuts and heavy quark masses, that the signal becomes comparable to the $\mathrm{Wjj}$ and $\mathrm{WW}$ processes for the SSC, and is one or two orders of magnitude smaller for the other colliders. Once one estimates the real six fermion backgrounds of the signals (see above) one concludes that signals are of the same 
order as the backgrounds for the Tevatron, UNK and LHC colliders, and dominate by $\sim 2$ orders of magnitude the background at the SSC. The main reason for larger background/signal ratios at UNK and LHC is the more relaxed cuts (compared to the ones required at the other colliders). We made these choices for illustration, without optimizing cuts. More stringent cuts on $p_{\mathrm{t}}$ would greatly reduce these ratios and, to some extent, also the signals [31].

Since the capability of hadron colliders for efficiently producing heavy quark pairs has been established, we discuss how to determine the masses of the particles involved and their character. The first indication of a new quark is a measured cross section larger than expected from the standard model sources of $\ell \nu \mathrm{jjjj}$ events. Q $\overline{\mathrm{Q}}$ initiated processes would be identified (and the heavy quark mass measured) demanding

$$
M_{\mathrm{Q}} \simeq M_{\mathrm{W}(\ell \nu) j} \simeq M_{\mathrm{W}(\mathrm{jj}) \mathrm{j}},
$$

where one of the four jets will be associated with the lepton pair. (Only the transverse momentum of the neutrino is known through the missing transverse momentum measurement. Assuming that $\ell \nu$ comes from a real $\mathrm{W}$, the longitudinal momentum of the $\nu$ can be determined - up to a sign, and both possibilities have to be taken into account.) As we stated above the neutral-to-charged ratio in eq. (3.2) then allows us to distinguish vector-like and sequential quarks. Previously a similar analysis of the $\ell \bar{\ell}$ jjjj sample had to be performed, in particular one should require

$$
M_{\mathrm{Q}} \simeq M_{\mathrm{Z}(\ell \bar{\ell}) \mathrm{j}} \simeq M_{\mathrm{W}(\mathrm{ji}) \mathrm{j}} \text {. }
$$

Finally, for vector-like quarks one can look for a intermediate mass Higgs in the initial $(\ell \nu, \ell \bar{\ell}) \mathrm{jjjj}$ samples, relaxing eqs. (3.3) and (3.4) and requiring only

$$
M_{\mathrm{W}\left(\ell v^{\prime}\right) \mathrm{j}} \simeq M_{\mathrm{jjj}} \simeq M_{\mathrm{Z}(\ell \bar{\ell}) \mathrm{j}} \simeq M_{\mathrm{Q}}
$$

and then plotting the invariant mass of two of the three jets reconstructing $M_{\mathrm{Q}}$. The standard gauge boson peaks corresponding to $\mathrm{W}, \mathrm{Z}, \mathrm{H} \rightarrow \mathrm{q} \overline{\mathrm{q}}$ must be observable. A scatter plot of the hypothetical $M_{\mathrm{Q}}$ versus $M_{\mathrm{jj}}$ could be used to enhance signal/background, by selecting the regions that have a concentration for $M_{\mathrm{Q}}$ and have $M_{\mathrm{jj}}=M_{\mathrm{Z}}, M_{\mathrm{W}}$ or a concentration at any possible $M_{\mathrm{H}}$. Note that eq. (3.5) ensures the sample to be $\bar{t} \bar{t}$ background free and to have a small contamination of the other backgrounds we consider.

In addition, other samples, as for instance the samples with more than one $\nu$ (although they are harder to control) should be consistent with the results we could find. Also, if some $\ell \bar{\ell} \ell \bar{\ell}$ jj events with

$$
M_{\mathrm{Z}(\ell \bar{\ell}) \mathrm{j}}=M_{\mathrm{Z}(\ell \bar{\ell}) \mathrm{j}} \simeq M_{\mathrm{Q}}
$$

are found, a new determination of $M_{\mathrm{Q}}$ is obtained. 


\subsection{VECTOR-LIKE LEPTONS}

Vector-like lepton cross sections are smaller than the vector-like quark ones at hadron colliders, because leptons are mainly produced by Drell-Yan fusion. This translates into a smaller upper bound on the accessible vector-like masses.

Once a $\bar{L} \overline{\mathrm{L}}$ pair has been produced, its signals involve two leptons plus either four extra jets, or two extra leptons and two jets, or four additional leptons, depending on the decay channels of the two bosons into which each initial lepton decays. The decay fractions for a singlet $E$ and a doublet $\left(\begin{array}{l}N \\ E\end{array}\right)$ into signals including at most one neutrino are given in table $9 \mathrm{a}$. In parentheses we give the fraction of Higgs mediated events. We have approximated the $\mathrm{W} / \mathrm{Z} / \mathrm{H}$ branching ratios to $2 / 1 / 1$ for the $\mathrm{E}$ singlet, although the values quoted in table 6 oscillate for the illustrative cases we consider, especially for the choices of masses near threshold. Within such an approximation, the relative gauge boson content in $E \bar{E}$ events is the same as for the $\mathrm{D} \overline{\mathrm{D}}$ case, given in eq. (3.1). In the doublet case, $\mathrm{N}$ always decays into $\mathrm{W} \ell$ and $\mathrm{E}$ into either $\mathrm{Z} \ell$ or $\mathrm{H} \ell$ with rates given in table 6 . We approximate the $\mathrm{Z} / \mathrm{H}$ ratio to one for table 9. In this case the relative total gauge boson content depends on the production cross section (table 5) because, in contrast with the quark case, they are flavour dependent. In table $9 \mathrm{~b}$ we give the total cross sections (with no cuts) for the different signals and colliders we consider. The $\ell \bar{\ell} \ell \nu \mathrm{jj}, \ell \bar{\ell} \mathrm{jjjj}$ and $\ell \nu \mathrm{jjjj}$ are the biggest ones. Let us discuss the $\ell \bar{\ell}$ jjjj signal first. The lepton pair does not come from a common source. In fact $\ell(\bar{\ell})$ originates from $\mathrm{L}(\overline{\mathrm{L}})$, so their joint invariant mass is not restricted to be close to $M_{\mathrm{Z}}$. This simple characteristic allows us to distinguish such 2 lepton +4 jet signal from many of the possible backgrounds (vector-like quarks included!). These contain either $\ell \bar{\ell}$ pairs from $\mathrm{Z}$ decays or from $\mathrm{WW} \rightarrow \ell \bar{\ell} \nu \nu$ where missing $p_{\mathrm{t}}$ is present and gives a distinctive signature from the one we are considering. (The same argument can be formulated saying that the two leptons resulting from the two-body decays of the new heavy leptons, are often back to back (in the transverse plane) as has been emphasized [32] for standard heavy leptons, in contradistinction with possible backgrounds from $\mathrm{W}$ and/or $\mathrm{Z}$ production). We have not performed a careful analysis of the background contribution to the $\ell \bar{\ell} \mathrm{q} \bar{q} q \bar{q}$ signal, but we believe that they have to be very small when $m_{\ell \bar{\ell}}$ is far from $M_{Z}$. (Another possibility for producing $\ell \bar{\ell}$ with $m_{\ell \bar{\ell}} \neq M_{Z}$ would be processes with 4 leptons where two of them escape detection. However, their contributions are expected to be small.) Thus, in order to look for a heavy lepton in the $\ell \bar{\ell}$ jjjj sample, with all final leptons and jets satisfying the cuts on pseudorapidity and transverse momentum (similarly as for quarks in subsect. 3.1), one demands the lepton pair mass to be somewhat larger than $m_{Z}$ in order to reject all $Z$ contributions. The remaining events should be analyzed looking for one lepton-two jet associations such that

$$
M_{\ell \mathrm{j}}=M_{\bar{\ell}_{\mathrm{jj}}} \simeq M_{\mathrm{L}},
$$

$M_{\mathrm{L}}$ being the measured mass of the reconstructed heavy lepton. The expected 
F. del Aguila et al. / Higgs production

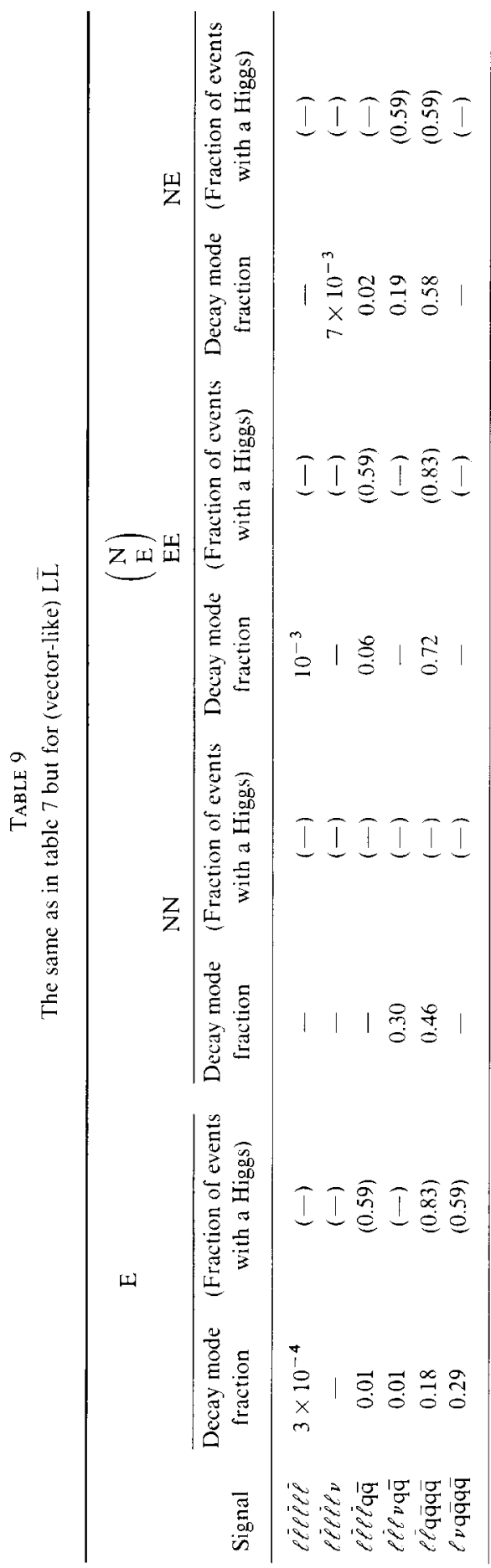

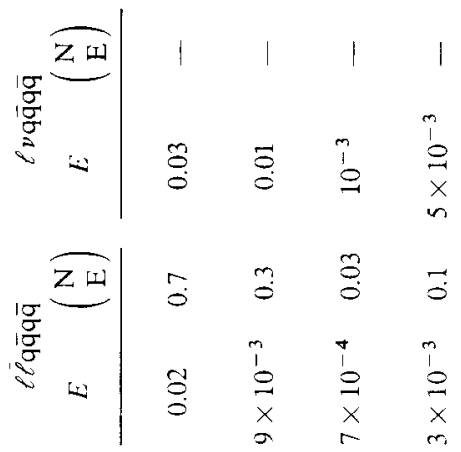

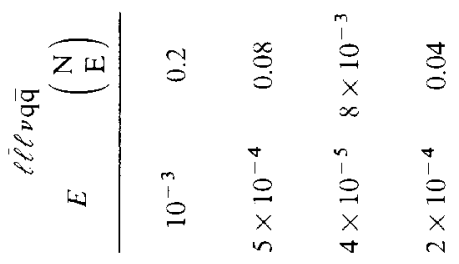

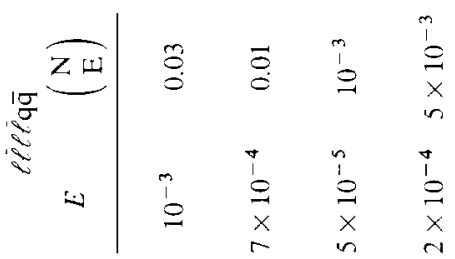

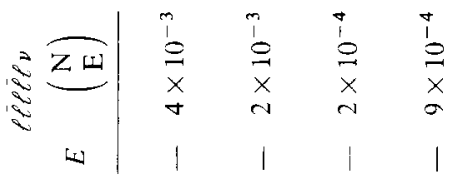

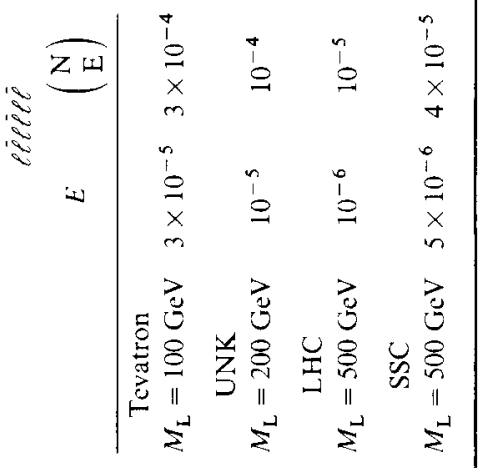


TABLE 10

Expected number of events (for the luminosities in table 2) for the dominant $L \bar{L}$ decay signals. All final fermions satisfy transverse momentum, $p_{\mathrm{t}}$, and pseudorapidity (except for the neutrino), $\eta$, cuts. We also cut on the invariant mass of the two leptons $M_{\ell \bar{\ell}, \ell \nu}$ to enforce that they do not originate from a $Z$ or W decay. (For the $\ell \bar{\ell} \ell \nu \mathrm{jj}$ signal see the text)

\begin{tabular}{|c|c|c|c|c|c|}
\hline & & $\begin{array}{c}\text { Tevatron } \\
\left(M_{\mathrm{L}}=100 \mathrm{GeV}\right) \\
|\eta|<4 \\
p_{\mathrm{t}}>10 \mathrm{GeV} \\
\text { No } M_{\ell \bar{\ell}, \ell \nu} \text { cut }\end{array}$ & $\begin{array}{c}\text { UNK } \\
\left(M_{\mathrm{L}}=200 \mathrm{GeV}\right) \\
|\eta|<4 \\
p_{\mathrm{t}}>10 \mathrm{GeV} \\
M_{\ell \ell, \ell \nu}>100 \mathrm{GeV}\end{array}$ & $\begin{array}{c}\text { LHC } \\
\left(M_{\mathrm{L}}=500 \mathrm{GeV}\right) \\
|\eta|<4 \\
p_{\mathrm{t}}>50 \mathrm{GeV} \\
M_{\ell \ell, \ell \nu}>200 \mathrm{GeV}\end{array}$ & $\begin{array}{c}\text { SSC } \\
\left(M_{\mathrm{L}}=500 \mathrm{GeV}\right) \\
|\eta|<4 \\
p_{\mathrm{t}}>50 \mathrm{GeV} \\
M_{\ell \dot{\ell}, \ell \nu}>200 \mathrm{GeV}\end{array}$ \\
\hline \multirow[t]{2}{*}{$\ell \bar{\ell} \mathrm{jjjj}$} & $\mathrm{E}$ & 0.01 & 0.5 & 1 & 5 \\
\hline & $\left(\begin{array}{l}\mathrm{N} \\
\mathrm{E}\end{array}\right)$ & 0.9 & 18 & 54 & 239 \\
\hline \multirow[t]{2}{*}{$\ell \nu \mathrm{jjjj}$} & $\mathrm{E}$ & 0.02 & 0.9 & 2 & 9 \\
\hline & $\left(\begin{array}{l}\mathrm{N} \\
\mathrm{E}\end{array}\right)$ & - & - & - & - \\
\hline \multirow[t]{2}{*}{$\ell \bar{\ell} \ell \nu \mathrm{jj}$} & $\mathrm{E}$ & $7 \times 10^{-4}$ & 0.03 & 0.08 & 0.3 \\
\hline & $\left(\begin{array}{l}\mathrm{N} \\
\mathrm{E}\end{array}\right)$ & 0.4 & 5 & 17 & 72 \\
\hline
\end{tabular}

number of events for the different vector-like leptons and hadron colliders considered (using the luminosities quoted in table 2) are given in table 10 for a given choice of the heavy lepton mass, and the cuts indicated. Standard sequential heavy leptons have similar cross sections and signals. However, they can be distinguished from the vector-like ones with a good reconstruction of the mass of the two jets $M_{\mathrm{ji}}$ accompanying the lepton [see eq. (3.7)]. If a sizable number of events have $m_{\mathrm{ij}} \simeq m_{Z}$ (E singlets decay into a $Z 64 \%$ of the time, and $\left(\begin{array}{l}N \\ E\end{array}\right)$ doublets do it often), we would have a clear indication of the vector-like nature of the leptons. (Standard ones only decay into $\mathrm{W}$, through charged currents.)

A similar analysis must be done for $\ell \nu \mathrm{jjjj}$ once $M_{\mathrm{L}}$ is known (from the $\ell \bar{\ell} \mathrm{jjjj}$ analysis). Events satisfying

$$
M_{\ell \mathrm{jj}} \simeq M_{\nu \mathrm{ji}} \simeq M_{\mathrm{L}}
$$

would establish the singlet vector-like nature of the heavy leptons (see table 10). In this case the primary $\mathrm{E}$ decay gives $\nu \mathrm{W}$ with subsequent boson decay into $\mathrm{q} \overline{\mathrm{q}}$, so the jet pair associated to the $\nu$ must fulfill $M_{\mathrm{j} j} \simeq M_{\mathrm{W}}$.

Finally the signal $\ell \bar{\ell} \ell \nu \mathrm{jj}$ can be analyzed in a similar way and used to check the consistency of the results obtained from the other channels.

Vector-like fermions are, like vector-like quarks, a source of Higgs production, which shows up via the invariant mass of the two-jet pairs accompanying the standard final leptons (except for the $\ell \bar{\ell} \ell \nu \mathrm{jj}$ channel and for the singlet E). The quoted fractions of events with a Higgs in table $9 \mathrm{a}$ were estimated assuming an intermediate Higgs mass and that $\mathrm{H} \rightarrow \mathrm{q} \overline{\mathrm{q}}$ with a branching ratio equal 1 . 
A final comment is devoted to the $\tau$ lepton. Our analysis for $\mathrm{L}$ decays relies on a good lepton $(e, \mu)$ identification. However, if the mixing parameters were such that $m_{3}^{\left({ }^{\prime}\right)} \gg m_{1,2}^{(\prime)}$, the $\tau$ lepton would be copiously produced in $\mathrm{L}$ decays. If it could not be efficiently identified, a more careful (and involved) analysis would be required and our conclusions should be revised.

\section{Conclusions and generalizations}

We have discussed vector-like fermion production at hadron colliders, and its relevance for the detection of the standard Higgs boson. We have concentrated on the intermediate mass region, $M_{\mathrm{H}}<2 M_{\mathrm{W}}$, where the standard Higgs detection is more problematic [34]. In table 11 we give the number of events with such a Higgs $(\mathrm{H} \rightarrow \mathrm{q} \overline{\mathrm{q}})$ for the different vector-like fermion masses, signals, hadron colliders and cuts considered in sects. 2 and 3. In particular, we conclude that if a new vector-like fermion with a mass $M_{\mathrm{F}} \leqslant 1 \mathrm{TeV}$ exists, a standard Higgs in the intermediate mass region cannot escape detection at the SSC. If $M_{\mathrm{H}}>2 M_{\mathrm{W}}$ the number of events with a Higgs in the $F \bar{F}$ sample is similar to those quoted in table 11 , but gives signals with 8 (or 10) final fermions. The jet pair from a Higgs in table 11 must be replaced by the four fermions coming from $\mathrm{H} \rightarrow W W, Z Z$. For this range of heavy Higgs masses, however, direct pair gauge boson production mediated by a Higgs will eventually give better signals [35]. At any rate, the $\vec{F} \vec{F}$ should also be observable. In this case (and also in the case of an intermediate mass Higgs if established in some other way [34]) the absence of such events would bound $M_{F}$. (Although the best way for establishing the existence of a new fermion is looking at its $W$ and $Z$ decays.) If a heavy vector-like fermion is found, and no decay $\mathrm{F} \rightarrow \mathrm{H}+\mathrm{f}$ is found, any model of the Higgs sector is very constrained.

A large part of our work has been devoted to the detection of a vector-like fermion, a step which should precede or be taken simultaneously with the Higgs search we propose. Vector-like fermions have masses unrelated to the standard model symmetry breaking and are therefore unbounded. They can be distinguished from a sequential heavy fermion because they have tree level flavour changing neutral currents [11]. They decay into Wf, Zf and Hf (f being a standard fermion) with similar frequency. In fact, the corresponding couplings are proportional. Some of the signals, however, coincide for a vector-like and a new sequential fermion. In these cases they are backgrounds of each other. With appropriate cuts the signal to (other) background ratios are larger than one; for distinguishing between vector-like and sequential fermion production we must compare between different final signatures. Looking to the $\ell \bar{\ell}$ jjjj, $\ell \nu \mathrm{jjjj}$ six final fermion signals and cutting on pseudorapidity and transverse momentum and demanding $W$ and/or $Z$ reconstruction we can reduce the backgrounds below the signals for $\mathrm{Q} \overline{\mathrm{Q}}$ production, allowing for a measurement of $M_{\mathrm{Q}}$. (For instance, the cuts in table 11 are appropriate for the Tevatron and the SSC, but the cuts for the other colliders are not stringent enough). 
TABLE 11

Expected number of events for the dominant $\bar{F} \bar{F}$ signals in the Higgs channel, with the same cuts as in tables 8 and 10

\begin{tabular}{|c|c|c|c|c|}
\hline & & $\ell \nu \mathrm{jijj}$ & $\ell \bar{\ell}_{\mathrm{jjj \textrm {j }}}$ & $\ell \bar{\ell} \ell \nu \mathrm{jj}$ \\
\hline \multicolumn{5}{|c|}{ Tevatron } \\
\hline$M_{\mathrm{Q}}=150 \mathrm{GeV}$ & $\mathrm{D}=\mathbf{U}$ & & & \\
\hline$|\eta|<4$ & & 4 & 0.6 & - \\
\hline$p_{\mathrm{t}}>10 \mathrm{GeV}$ & $=\frac{1}{2}\left(\begin{array}{l}\mathrm{U} \\
\mathrm{D}\end{array}\right)$ & & & \\
\hline $\begin{array}{c}M_{\mathbf{L}}=100 \mathrm{GcV} \\
|\eta|<4\end{array}$ & $\mathrm{E}$ & 0.01 & $9 \times 10^{-3}$ & - \\
\hline$p_{1}>10 \mathrm{GeV}$ & $\left(\begin{array}{l}\mathrm{N} \\
\mathrm{E}\end{array}\right)$ & - & 0.3 & 0.07 \\
\hline \multicolumn{5}{|c|}{ UNK } \\
\hline $\begin{array}{c}M_{\mathrm{Q}}=300 \mathrm{GeV} \\
|\eta|<4\end{array}$ & $\mathrm{D}=\mathrm{U}$ & 32 & 5 & - \\
\hline$p_{\mathrm{t}}>10 \mathrm{GeV}$ & $=\frac{1}{2}\left(\begin{array}{l}U \\
D\end{array}\right)$ & & & \\
\hline $\begin{array}{c}M_{\mathrm{L}}=200 \mathrm{GeV} \\
|\eta|<4\end{array}$ & E & 0.5 & 0.5 & - \\
\hline$p_{t}>10 \mathrm{GeV}$ & $\left(\begin{array}{l}N \\
E\end{array}\right)$ & - & 10 & 2 \\
\hline \multicolumn{5}{|l|}{$M_{\ell \ell, \ell \nu}>100 \mathrm{GeV}$} \\
\hline \multicolumn{5}{|c|}{ LHC } \\
\hline $\begin{array}{c}M_{\mathrm{Q}}=700 \mathrm{GeV} \\
|\eta|<4\end{array}$ & $\mathrm{D}=\mathbf{U}$ & 196 & 30 & - \\
\hline$p_{\mathrm{t}}>50 \mathrm{GeV}$ & $=\frac{1}{2}\left(\begin{array}{l}U \\
D\end{array}\right)$ & & & \\
\hline $\begin{array}{c}M_{\mathrm{L}}=500 \mathrm{GeV} \\
|\eta|<4\end{array}$ & $\mathrm{E}$ & 1 & 1 & - \\
\hline$p_{1}>50 \mathrm{GeV}$ & $\left(\begin{array}{c}\mathrm{N} \\
\mathrm{E}\end{array}\right)$ & - & 31 & 6 \\
\hline \multicolumn{5}{|l|}{$M_{\ell \ell, \ell \nu}>200 \mathrm{GeV}$} \\
\hline \multicolumn{5}{|c|}{$\mathrm{SSC}$} \\
\hline $\begin{array}{c}M_{\mathrm{Q}}=700 \mathrm{GeV} \\
|\eta|<4\end{array}$ & $\mathrm{D}=\mathrm{U}$ & 699 & 107 & - \\
\hline$p_{\mathrm{t}}>10 \mathrm{GeV}$ & $=\frac{1}{2}\left(\begin{array}{l}\mathbf{U} \\
\mathbf{D}\end{array}\right)$ & & & \\
\hline $\begin{array}{c}M_{\mathrm{L}}=500 \mathrm{GeV} \\
|\eta|<4\end{array}$ & $\mathrm{E}$ & 5 & 4 & - \\
\hline$p_{\mathrm{t}}>50 \mathrm{GeV}$ & $\left(\begin{array}{l}N \\
E\end{array}\right)$ & - & 133 & 28 \\
\hline$M_{e \dot{\ell}, \ell_{\nu}}>200 \mathrm{GcV}$ & & & & \\
\hline
\end{tabular}


For leptons an extra cut on the invariant mass of the two leptons produced in the initial two-body decays (the only two leptons in the dominant signals) is required to get rid of the large backgrounds resulting from $\mathrm{Z} \rightarrow \ell \bar{\ell}$ and $\mathrm{W} \rightarrow \ell \nu$ production, $M_{\ell \bar{\ell}, \ell \nu} \gg M_{\mathrm{Z}}$. Having established the existence of a new heavy fermion, to distinguish between a vector-like and a sequential one we must compare charged and neutral decay processes. Finally, looking at the two jet invariant mass of the three partons reconstructing $M_{\mathrm{F}}$ (for $M_{\mathrm{H}}<2 M_{\mathrm{W}}$ ) in the complete $\bar{F} \overline{\mathrm{F}}$ sample, the Higgs can be detected and its mass $M_{\mathrm{H}}$ measured.

Our numerical results were rough estimates because, in particular, we worked at the parton level and neglected QCD corrections, with no proper background estimates, without detector simulation, etc.... We did not discuss extreme cases where the mixing parameters are chosen to force the new vector-like quark (lepton) to decay mainly into a heavy top $(\tau)$. In view of the numerical results we believe that our approximations are sufficient to have a good grasp of the main physics.

Vector-like leptons have cross sections too small for detection at present hadron colliders, whereas a relative light vector-like quark $\left(M_{\mathrm{Q}}>M_{\mathrm{W}}\right)$ could be detectable at the Tevatron (but not at the Spps). Larger hadron colliders, in particular the $\mathrm{SSC}$, have more chances of discovering the presence of heavy (vector-like) fermions.

We have discussed the simplest extensions of the standard model with extra vector-like fermions. If more than one heavy vector-like fermion exists, the analysis still holds. The mass terms invariant under the standard model symmetries (which are the main source of vector-like fermion masses) can be diagonalized without any loss of generality, and what we discussed above applies to each mass eigenstate of the vector-like fermions. Each vector-like family will have its own mass and mixings with the standard fermions. If the new replicas were degenerate in mass, their cross sections would add (in particular, for three families, the cross sections would be three times larger [12]). In models with an extended and complicated Higgs sector the details may be different (as in the case of supersymmetric models, which are under consideration). A fourth sequential family and/or large $m^{(\prime)} / M_{\mathrm{F}}$ mixings may invalidate our approximations (for we only keep leading terms in $m^{(2)} / M_{\mathrm{F}}$ ) and part of our conclusions.

Our basic purpose is simply to alert experimenters to the new opportunities to explore the Higgs sector should vector-like quarks (leptons) exist. If they do exist, the Tevatron collider becomes a Higgs boson factory instead of a machine where low production rates and difficult signatures render it essentially useless for Higgs bosons, and at the SSC it becomes far easier to find intermediate-mass Higgs bosons.

We thank C. Albajar, U. Baur, L. Di Lella, K. Jakobs, R. Kleiss and M. Quirós for clarifying discussions. Two of us (F.A. and L1.A.) acknowledge the kind hospitality of the CERN Theory Group. 


\section{References}

[1] P. Ramond, in Proc. 4th Kyoto summer Institute on Grand unified theories and related topics (Kyoto, Japan, 1981), ed. M. Konuma and T. Maskawa (World Science, Singapore, 1981);

Standard model vector-like leptons were used (but with very low masses) by T.P. Cheng and L.F. Li, Phys. Rev. D16 (1977) 1429

[2] F. del Aguila and M.J. Bowick, Phys. Lett. B119 (1982) 142

[3] F. del Aguila and M.J. Bowick, Nucl. Phys. B224 (1983) 107

[4] P. Fishbane, K. Gamers, S. Meshkov and R. Norton, Phys. Rev. D32 (1985) 1189;

P. Fishbane, R. Norton and M. Rivard, Phys. Rev. D33 (1986) 2632

[5] R.W. Robinett, Phys. Rev. D33 (1986) 1908;

V. Barger, N. Deshpande, R.J.N. Phillips and K. Whisnant, Phys. Rev. D33 (1986) 1912;

M.J. Duncan and P. Langacker, Nucl. Phys. B277 (1986) 285:

S.M. Barr, Phys. Rev. Lett. 55 (1986) 2778;

J.A. Grifols, A. Méndez and J. Solà, Phys. Rev. Lett. 57 (1986) 2348;

J.L. Hewett and T.G. Rizzo, Z. Phys. C34 (1987) 49;

B. Mukhopadhyaya, A. Ray and A. Raychaudhuri, Phys. Lett. B186 (1987) 147

[6] J. Bernabéu, A. Santamaria, J. Vidal, A. Méndez and J.W.F. Valle, Phys. Lett. B187 (1987) 303;

P. Langacker and D. London, Phys. Rev. D38 (1988) 886, 907

[7] W. Buchmüller and M. Gronau, Phys. Lett. B220 (1989) 641;

R. Barbieri and J. Hall, Nucl. Phys. B319 (1989) 1;

T.G. Rizzo. University of Wisconsin-Madison preprint, MAD/PH/480, 1989;

M.J. Duncan, CERN preprint, CERN-TH.5429/89

[8] F. del Aguila and J. Cortés, Phys. Lett. B156 (1985) 243;

F. del Aguila. M.K. Chase and J. Cortés, Nucl. Phys. B271 (1986) 61

[9] F. del Aguila, G.L. Kane and M. Quirós, Phys. Lett. B196 (1987) 531:

J. Vidal, Phys. Rev. D38 (1988) 865;

K.S. Babu and L. Roszkowski, Nucl. Phys. B317 (1989) 97

[10] B. Allés, Phys. Lett. B221 (1989) 343

[11] G.L. Kane and M. Peskin, Nucl. Phys. B195 (1982) 29

[12] F. del Aguila, G.L. Kane and M. Quirós, Phys. Rev. Lett. 63 (1989) 942

[13] F. del Aguila, E. Laerman and P. Zerwas, Nucl. Phys. B297 (1988) 1

[14] V. Barger, N.G. Deshpande, W.Y. Keung, M.H. Reno and M. Ruiz-Altaba, Mod. Phys. Lett. A2 (1987) 437

[15] I. Gürsey, P. Ramond and P. Sikivie, Phys. Lett. B60 (1976) 177;

Y. Achiman and B. Stech, Phys. Lett. B77 (1978) 389;

Q. Shafi, Phys. Lett. B79 (1978) 301;

H. Ruegg and T. Schücker, Nucl. Phys. B161 (1979) 388;

R. Barbieri and D.V. Nanopoulos, Phys. Lett. B91 (1980) 369

[16] B.L. Combridge, Nucl. Phys. B151 (1979) 489

[17] E. Eichten, I. Hinchliffe, K. Lane and C. Quigg, Rev. Mod. Phys. 56 (1984) 579; (E) 58 (1986) 1065. and references therein

[18] S. Dawson and S.S.D. Willenbrock, Nucl. Phys. B284 (1987) 449

[19] C. Albajar et al. (UA1 Collaboration), Z. Phys. C37 (1988) 489, 505; Phys. Lett. B213 (1988) 405;

L. Di Lella. private communication

[20] D. Duke and J. Owens, Phys. Rev. D30 (1984) 49

[21] A.D. Martin, R.G. Roberts and W.J. Stirling, Phys. Rev. D37 (1988) 1161

[22] P. Nason, S. Dawson and R.K. Ellis, Nucl. Phys. B303 (1988) 607;

G. Altarelli, M. Diemoz, G. Martinelli and P. Nason, Nucl. Phys. B308 (1988) 724;

W. Beenakker, H. Kuijf and W.L. van Neerven, Phys. Rev. D40 (1989) 54

[23] S.S.D. Willenbrock and D.A. Dicus, Phys. Lett. B156 (1985) 429

[24] Particle Data Book, M. Aguilar-Benítez et al., Phys. Lett. B204 (1988) 1

[25] H. Baer, V. Barger and R.J. Phillips, Phys. Rev. D32 (1985) 688 
[26] F.A. Berends, W.T. Giele and H. Kuijf, Leiden University preprint, 1989;

S.J. Parke and T.R. Taylor, Phys. Rev. Lett. 56 (1986) 2459

[27] W.J. Stirling, R. Kleiss and S.D. Ellis, Phys. Lett. B163 (1985) 261;

J.F. Gunion, Z. Kunszt and M. Soldate, Phys. Lett. B163 (1985) 389; (E) B168 (1986) 427

[28] F.A. Berends, W.T. Giele, H. Kuijf, R. Kleiss and W.J. Stirling, Phys. Lett. B224 (1989) 237;

K. Hagiwara and D. Zeppenfeld, Nucl. Phys. B313 (1989) 560

[29] R.W. Brown and K.O. Mikaclian. Phys. Rev. D19 (1979) 922

[30] U. Baur, E.W.W. Glover and J.J. van der Bij, Nucl. Phys. B318 (1989) 106

[31] F. del Aguila, Ll. Ametller, R.D. Field and L1. Garrido, Phys. Lett. B201 (1988) 375: B221 (1989) 408

[32] I. Hinchliffe, Int. J. Mod. Phys. A4 (1989) 3867; see also V. Barger, T. Han and J. Ohnemus, Phys. Rev. D37 (1988) 1174

[33] H. Baer, V. Barger, H. Goldberg and J. Ohnemus, Madison preprint, MAD/PH/427;

H. Baer, V. Barger and R.J.N. Phillips, Phys. Rev. D39 (1989) 2809; 3310; Madison preprint $\mathrm{MAD} / \mathrm{PH} / 458$ :

J.L. Rosner, Phys. Rev. D39 (1989) 3297

[34] J.F. Gunion, G.L. Kane and J. Wudka, Nucl. Phys. B229 (1988) 231

[35] J.F. Gunion, H.E. Haber, G.L. Kane and S. Dawson, The physics of Higgs bosons, UCD-89-4, SCIPP-88/11. BNL-41644, to be published 